\title{
ON THE REMAINDERS OF CERTAIN METRIC SPACES
}

\author{
BY
}

KENNETH D. MAGILL, JR.( $\left.{ }^{1}\right)$

To Orrin Frink on his 70th birthday

\begin{abstract}
Let $X$ be completely regular and Hausdorff. The family $\mathscr{R}(X)$ of all those spaces which are remainders of $X$ in compactifications has attracted a considerable amount of interest. In this paper we determine, in a certain sense, the family $\mathscr{R}(X)$ for a fairly large number of spaces $X$.
\end{abstract}

1. Statement of main theorem and a corollary. We state at the outset that all topological spaces under discussion here are assumed to be completely regular and Hausdorff. Moreover, a countable set in this paper is one which is in one-to-one correspondence with the natural numbers. In particular, finite sets will not be regarded as being countable. A remainder of a space $X$ is any space of the form $\alpha X-X$ where $\alpha X$ is a compactification of $X$. For any space $X$, we use the symbol $\mathscr{R}(X)$ to denote the family of all remainders of $X$. In other words, $\mathscr{R}(X)$ consists precisely of those spaces with which $X$ can be compactified. The main result of this paper shows that, for many spaces $X, \mathscr{R}(X)$ consists of all continuous images of the free union of a finite number of copies of a familiar space. We recall that the free union of a family of spaces $\left\{X_{n}: n \in \Lambda\right\}$ is the set $\bigcup\left\{X_{n}: n \in \Lambda\right\}$ topologized by defining $G \subset \bigcup\left\{X_{n}: n \in \Lambda\right\}$ to be open if and only if $G \cap X_{n}$ is open for each $n \in \Lambda$. We use the symbol $R^{+}$to denote the space of nonnegative real numbers and, as is customary, the Stone-Čech compactification of a space $X$ will be denoted by $\beta X$. Now we are in a position to state the main

THEOREM. Suppose $X$ is noncompact, locally compact, locally connected and metric and $\mathscr{R}(X)$ contains no countable spaces. Then there exists a positive integer $N$ such that $\mathscr{R}(X)$ consists precisely of all continuous images of the free union of $N$ copies of $\beta R^{+}-R^{+}$.

We denote the cardinality of any set $X$ by card $X$. In [4, p. 91], it is shown that card $\beta R=$ card $\beta N$ where $R$ denotes the space of real numbers and $N$ denotes the

Received by the editors May 18, 1970 and, in revised form, October 14, 1970.

AMS 1969 subject classifications. Primary 5453.

Key words and phrases. Compactifications, remainders of spaces in compactifications.

( $\left.{ }^{1}\right)$ Some of the work on this paper was done while the author was visiting the Institute of Advanced Studies of the Australian National University. He takes this opportunity to express his appreciation to his friends and colleagues there for making the visit a most beneficial and enjoyable one. Financial support from the Institute and also from the research foundation of the State University of New York is gratefully acknowledged.

Copyright (C) 1971, American Mathematical Society 
countable discrete space. Very similar techniques yield the fact that card $\beta R^{+}$ $=$ card $\beta N$. Since card $\beta N=2^{c}$ (see [4, p. 131]) where $c$ denotes the cardinality of the continuum, we obtain from the previous theorem the following

COROllary. Suppose $X$ is noncompact, locally compact, locally connected and metric and $\mathscr{R}(X)$ contains no countable spaces. Then card $K \leqq 2^{c}$ for each remainder $K$ of $X$.

The conclusion of the corollary and hence that also of the theorem need not hold if one does not require that $\mathscr{R}(X)$ contain no countable spaces. To see this, let $K$ be any compact space with card $K>2^{c}$ and let $X$ be any discrete space with card $X$ $\geqq$ card $K$. Then there exists a (necessarily continuous) function $f$ from $X$ onto $K$ such that $f^{-1}(y)$ is noncompact (in this case, infinite) for each $y \in K$. Therefore, it follows from Lemma 2 of [10] that $K$ is a remainder of $X$. This establishes the fact that one needs to require that $\mathscr{R}(X)$ contain no countable spaces since $X$ is noncompact, locally compact, locally connected and metric.

Perhaps it would be well at this point to mention how one can determine whether or not $\mathscr{R}(X)$ contains a countable space, that is, if $X$ has a countable compactification. The paper [8] is devoted to this problem and gives several characterizations of locally compact spaces which have countable compactifications (e.g., Theorem (2.1), p. 616, and Corollary (2.4), p. 620). Furthermore, Corollary (2.2) of [8, p. 619] states that if $X$ is locally compact and there exists a positive integer $N$ such that every compact subset of $X$ is contained in a compact subset whose complement consists of at most $N$ components, then $\mathscr{R}(X)$ contains no countable spaces.

It is appropriate now to express our appreciation to the referee for his helpful comments. In particular, we are indebted to him for Lemma 2 of this paper which extends Theorem 8.6 of [3, p. 247].

2. Proof of the theorem. Throughout this section, the symbol $X$ will be reserved to denote the space of the theorem. That is, $X$ is noncompact, locally compact, locally connected and metric and $\mathscr{R}(X)$ contains no countable spaces. It seems to be convenient to accomplish the proof through a sequence of lemmas. We begin with

\section{LEMMA 1. The space $X$ is second countable.}

Proof. Since $X$ is metric, it is sufficient to show that it is Lindelöf [3, Theorem 5.6, p. 187]. First, we show that $X$ has only finitely many components. Suppose the contrary is true. Since $X$ is locally connected, each component is open and it follows from Corollary (2.5) of $[8$, p. 620] that $X$ has a countable remainder which, of course, is a contradiction.

Thus, we have $X=\bigcup\left\{Y_{i}\right\}_{i=1}^{M}$ where each $Y_{i}$ is a component of $X$. Now each $Y_{i}$ is a locally compact, connected metric space and is therefore $\sigma$-compact [3, Theorem 7.3, p. 241]. Hence, $X$ is $\sigma$-compact and is therefore Lindelöf. 
Since $\mathscr{R}(X)$ contains no countable spaces, it follows from Theorem (2.1) of $[8$, p. 616] and Corollary $(2.5)$ of $[7$, p. 1078] that there exists a positive integer $N$ such that $X$ has an $N$-point compactification but no $M$-point compactification for $M>N$. We let $\alpha X$ denote the $N$-point compactification of $X$ and we denote the $N$-points of $\alpha X-X$ by $\left\{\infty_{i}\right\}_{i=1}^{N}$.

It is proven in [3, Theorem 8.6, p. 247] that the one-point compactification of a locally compact metric space is metrizable if and only if the space is second countable. We need the analogous result for $N$-point compactifications and this can be proved in much the same way. However, this is also an immediate consequence of the following lemma which is due to the referee. As far as we know, this result has not appeared before so it seems appropriate to include it here.

LEMMA 2. Let $X$ be locally compact and let $\gamma X$ be any compactification of $X$ with metrizable remainder. Then $\gamma X$ is metrizable if and only if $X$ is second countable.

Proof. We first prove sufficiency. Since $X$ is locally compact, $\gamma X-X$ is compact and therefore second countable. Consequently, there exists a homeomorphism $h$ from $\gamma X-X$ into the Hilbert cube $C$. Since $C$ is an absolute retract, $h$ can be continuously extended to a function $h^{*}$ which maps $\gamma X$ into $C$. Now define a function $k$ mapping $\gamma X$ into the one-point compactification $\hat{X}=X \cup\{\infty\}$ of $X$ by

$$
\begin{array}{ll}
k(x)=x & \text { for } x \in X, \\
k(x)=\infty & \text { for } x \in \gamma X-X .
\end{array}
$$

Then $k$ is continuous and it follows that the function $t$ mapping $\gamma X$ into $C \times \hat{X}$ which is defined by

$$
t(x)=\left(h^{*}(x), k(x)\right) \text { for } x \in \gamma X
$$

is continuous. In fact, one readily shows that $t$ is injective and, since $\gamma X$ is compact, it follows that $t$ is an embedding. Since $X$ is second countable, Theorem 8.6 of [3, p. 247] assures us that $\hat{X}$ is metrizable. Thus $C \times \hat{X}$ is metrizable and it follows that $\gamma X$ is also.

As for the necessity portion of the proof, if $\gamma X$ is metrizable it must be second countable in view of the fact that it is compact. Consequently, $X$ is second countable.

From Lemmas 1 and 2, we immediately obtain

LEMMA 3. $\alpha X$ is a metric space.

Now we choose any $\infty_{i} \in \alpha X$ and prove

LEMmA 4. Let $G$ be any open subset of $\alpha X$ containing $\infty_{i}$. Then there exists a connected open neighborhood $H$ of $\infty_{i}$ such that $H \subset G$ and $H-\left\{\infty_{i}\right\}$ is also connected.

Proof. Since $X$ is second countable and locally compact, there exists, according to problem 19 of $[1$, p. 233], a metric $d$ which is compatible with the topology on $X$ and is such that the compact subsets are precisely the closed and bounded subsets 
of $X$. Now let $\left\{V_{j}\right\}_{j=1}^{N}$ be any family of mutually disjoint open subsets of $X$ such that $\infty_{j} \in V_{j}$ for each $j$ and $V_{i} \subset G$. Let $K=\alpha X-\bigcup\left\{V_{j}\right\}_{j=1}^{N}$ and let $V_{j}^{\prime}=V_{j}-\left\{\infty_{j}\right\}$. Each $K \cup V_{j}$ is the complement of $\bigcup\left\{V_{t}\right\}_{t \neq j}$ in $\alpha X$ and is therefore compact. Since $\infty_{j}$ is a limit point of $V_{j}$, it follows that $K \cup V_{j}^{\prime}$ is not compact for each $j$. Now let $\left\{W_{\alpha}: \alpha \in \Lambda\right\}$ denote the components of $V_{i}^{\prime}$. Since $V_{i}^{\prime}$ is locally connected, each $W_{\alpha}$ is open in $X$. We assert that

(4.1) $K \cup W_{\alpha_{0}}$ is not compact for some $\alpha_{0} \in \Lambda$.

The proof is by contradiction so accordingly, we suppose that $K \cup W_{\alpha}$ is compact for each $\alpha \in \Lambda$. Now choose any point $p \in K$ and for each positive integer $n$, let

$$
B_{n}=\{x \in X: d(x, p) \leqq n\} .
$$

Since the metric $d$ is such that closed bounded subsets of $X$ are compact, each $B_{n}$ is compact. Now $K \cup V_{i}^{\prime}$ is the complement in $X$ of $\cup\left\{V_{j}^{\prime}\right\}_{j \neq i}$ and is therefore closed in $X$. Consequently, since $K \cup V_{i}^{\prime}$ is not compact, it cannot be contained in any compact subset of $X$. Since each $K \cup W_{\alpha}$ is assumed to be compact, it follows that $W_{\alpha} \notin B_{1}$ for infinitely many $\alpha$. Choose two of these sets and denote them by $A_{1}$ and $C_{1}$ respectively. Since both $K \cup A_{1}$ and $K \cup C_{1}$ are compact, both $A_{1}$ and $C_{1}$ are bounded and hence lie entirely within $B_{n_{2}}$ for suitable $n_{2}>1$. Just as before, we find two components $A_{2}$ and $C_{2}$ of $V_{i}^{\prime}$ both of which have points that lie outside $B_{n_{2}}$. Continuing in this manner, we get a subsequence $\left\{B_{n_{j}}\right\}$ of $\left\{B_{n}\right\}$ and two sequences of sets $\left\{A_{j}\right\}$ and $\left\{C_{j}\right\}$ such that $A_{j} \notin B_{n_{j}}$ and $C_{j} \notin B_{n}$, for each positive integer $j$. Now let $E_{1}=\bigcup\left\{A_{j}\right\}_{j=1}^{\infty}$ and $E_{2}=V_{i}^{\prime}-E_{1}$. Then both $E_{1}$ and $E_{2}$ are unbounded open subsets of $X$ and it follows that neither of the sets $K \cup E_{1}$ and $K \cup E_{2}$ is compact. Thus, $\left\{V_{j}^{\prime}\right\}_{j \neq 1} \cup\left\{E_{1}, E_{2}\right\}$ is a mutually disjoint family of $N+1$ open subsets of $X$ with the property that the union of any one of these sets with $K$ is not compact. Consequently, Theorem (2.1) of [7, p. 1075] implies that $X$ has an $N+1$-point compactification which, of course, is a contradiction. This completes the verification of statement (4.1).

It now follows that $K^{*}=K \cup\left(V_{i}^{\prime}-W_{\alpha_{0}}\right)$ must be compact. If not, $\left\{V_{i}^{\prime}\right\}_{j \neq i}$ $\cup\left\{W_{\alpha_{0}}, V_{i}^{\prime}-W_{\alpha_{0}}\right\}$ is a mutually disjoint family of $N+1$ open subsets of $X$ such that the union of $K$ with any of these sets is not compact. As before, this would result in the contradiction that $X$ has an $N+1$-point compactification. Furthermore, since $\infty_{i}$ is a limit point of $V_{i}^{\prime}$ and $K^{*}$ is compact, it follows that $\infty_{i}$ must be a limit point of $W_{\alpha_{0}}$. Thus, $W_{\alpha_{0}} \cup\left\{\infty_{i}\right\}$, which we will denote by $H$, is connected. Now we note that $\left(\alpha X-K^{*}\right) \cap V_{i}$ is a neighborhood of $\infty_{i}$ which is contained in $G$. This proves the lemma since $H=\left(\alpha X-K^{*}\right) \cap V_{i}$ and $H-\left\{\infty_{i}\right\}=W_{\alpha_{0}}$ is connected.

Since $X$ is locally connected by hypothesis, we appeal to Lemma 4 and immediately get

LEMMA 5. $\alpha X$ is locally connected.

Now let $H_{1}$ be any connected open subset of $\alpha X$ which contains $\infty_{1}$ but none of the other $\infty_{j}$. By the theorem of Mazurkiewicz, Moore and Menger [6, Theorem 1, 
p. 254], $\alpha X$ is locally arcwise connected since it is both complete and locally connected. Since $H_{1}$ is an open subset of $\alpha X$, it too must be locally arcwise connected. Now choose any compact subset $W_{1} \subset H_{1}$ such that $\infty_{1}$ belongs to the interior of $W_{1}$. It follows from Theorem 5 of $[6$, p. 253] that there exists a Peano continuum $K_{1}$ such that $W_{1} \subset K_{1} \subset H_{1}$. Thus, we have proved the existence of a Peano continuum $K_{1}$ such that $\infty_{1}$ belongs to int $K_{1}$ (the interior of $K_{1}$ ) and $\infty_{j} \notin K_{1}$ for $j \neq 1$. We see that by continuing in much the same way, we can produce $N$ mutually disjoint Peano continua $\left\{K_{i}\right\}_{i=1}^{N}$ such that $\infty_{i} \in$ int $K_{i}$ for each $i$. We let $A_{i}=K_{i}-\left\{\infty_{i}\right\}$ and we prove

\section{LEMMA $6 . A_{i}$ is connected.}

Proof. Suppose $A_{i}$ is not connected. Then $A_{i}$ is the union of two nonempty disjoint closed subsets $B$ and $C$ of $A_{i}$. Since $A_{i} \cup\left\{\infty_{i}\right\}=K_{i}$ is connected, $\infty_{i}$ is a limit point of both $B$ and $C$. Otherwise, one of the sets, say $B$, would be closed in $K_{i}$. But this would result in the contradiction that $K_{i}$ is not connected since $B$ is also open in $K_{i}$. Since $\infty_{i} \in$ int $K_{i}$, there exists, according to Lemma 3, a connected open subset $H$ of $\alpha X$ such that $\infty_{i} \in H \subset K_{i}$ and $H-\left\{\infty_{i}\right\}$ is connected. Since $\infty_{i}$ is a limit point of both $B$ and $C$, it follows that $H \cap B \neq \varnothing \neq H \cap C$. But this implies that $H-\left\{\infty_{i}\right\}$ is not connected, which is a contradiction. Thus, $A_{i}$ must be connected.

LEMMA 7. $A_{i}$ has no two-point compactification.

Proof. Let $V$ be any compact subset of $A_{i}$ and let $\left\{W_{\alpha}: \alpha \in \Lambda\right\}$ denote the components of $A_{i}-V$. We assert that

(7.1) $V \cup W_{\alpha_{0}}$ is not compact for some $\alpha_{0} \in \Lambda$.

We prove statement (7.1) by contradiction. Assume each $V \cup W_{\alpha_{0}}$ is compact and, just as in the verification of statement (4.1) in the proof of Lemma 4, we construct two open sets $E_{1}$ and $E_{2}$ such that $E_{1} \cup E_{2}=A_{i}-V$ and neither of the sets $V \cup E_{1}$ nor $V \cup E_{2}$ is compact. Now int $K_{i}-V$ is an open subset of $\alpha X$ containing $\infty_{i}$ so there exists, by Lemma 4, a connected open neighborhood $H$ of $\infty_{i}$ such that $H \subset$ int $K_{i}-V$ and $H-\left\{\infty_{i}\right\}$ is also connected. Since $V$ is compact but neither $V \cup E_{1}$ nor $V \cup E_{2}$ is compact, it follows that $\infty_{i}$ is a limit point of both $E_{1}$ and $E_{2}$. Then $H \cap E_{1} \neq \varnothing \neq H \cap E_{2}$ which implies that $H-\left\{\infty_{i}\right\}$ $=\left(H \cap E_{1}\right) \cup\left(H \cap E_{2}\right)$ is not connected. This is a contradiction so we conclude that (7.1) is valid. Now let $W^{*}=\bigcup\left\{W_{\alpha}: \alpha \neq \alpha_{0}\right\}$ and let $V^{*}=V \cup W^{*}$. We assert that

(7.2) $V^{*}$ is compact.

An argument identical to that used above applies. If $V^{*}$ is not compact, then $\infty_{i}$ is a limit point of both $W_{\alpha_{0}}$ and $W^{*}$ which are both open sets since $A_{i}-V$ is locally connected. From this it follows that $H \cap W_{\alpha_{0}} \neq \varnothing \neq H \cap V^{*}$ which again results in the contradiction that $H-\left\{\infty_{i}\right\}=\left(H \cap W_{\alpha_{0}}\right) \cup\left(H \cap V^{*}\right)$ is not connected. Thus $V^{*}$ is compact and $A_{i}-V^{*}=W_{\alpha_{0}}$ is connected. We have shown that every compact subset of $A_{i}$ is contained in a compact subset whose complement is 
connected. We can now appeal to Theorem (2.6) of [7, p. 1079] and conclude that $A_{i}$ has no two-point compactification.

In view of Lemmas 6 and 7 and the fact that $A_{i}$ is a nonempty open subset of a Peano continuum, we may conclude that $A_{i}$ is noncompact, locally compact, connected, locally connected, metric and has no two-point compactification. But this is precisely the definition of a ringed space as given in [2, Definition (2.1)]. Therefore we have

Lemma 8. Each $A_{i}$ is a ringed space.

Before proceeding to the next lemma, it will be convenient to introduce some notation. The symbol $\bigvee\left\{Y_{i}\right\}_{i=1}^{N}$ will be used to denote the free union of the topological spaces $Y_{1}, Y_{2}, \ldots, Y_{N}$.

Lemma 9. $\beta X-X=\bigvee\left\{\beta A_{i}-A_{i}\right\}_{i=1}^{N}$.

Proof. By a well-known property of $\beta X$, there exists a continuous function $f$ mapping $\beta X$ onto $\alpha X$ such that $f(x)=x$ for each $x \in X$. Since $f$ maps $\beta X-X$ onto $\alpha X-X$ it follows that

$$
\beta X-X=\bigvee\left\{f^{-1}\left(\infty_{i}\right)\right\}_{i=1}^{N} .
$$

The proof will be complete when we show that $f^{-1}\left(\infty_{i}\right)=\beta A_{i}-A_{i}$, and for this, it is sufficient to show that $f^{-1}\left(K_{i}\right)=\beta A_{i}$. First we show that $A_{i}$ is dense in $f^{-1}\left(K_{i}\right)$ and this is where we need the fact that $\infty_{i}$ belongs to the interior (with respect to $\alpha X$ ) of $K_{i}$. Choose any $p \in f^{-1}\left(K_{i}\right)-A_{i}=f^{-1}\left(\infty_{i}\right)$ and let $V$ be any open subset of $\beta X$ which contains $p$. Then $f^{-1}$ (int $\left.K_{i}\right) \cap V$ is a nonempty open subset of $\beta X$ and therefore must contain a point $x$ of $X$. Since $f(x)=x$, it follows that $x \in$ int $K_{i} \cap V$ $\cap X \subset A_{i} \cap V$. Hence, $A_{i}$ is dense in $f^{-1}\left(K_{i}\right)$ and since $f^{-1}\left(K_{i}\right)$ is compact, we conclude that it is a compactification of $A_{i}$. To show that it is actually $\beta A_{i}$, it is sufficient to show that any bounded continuous real-valued function $g$ on $A_{i}$ can be continuously extended over $f^{-1}\left(K_{i}\right)$ and this is easily accomplished. Since $A_{i}$ is a closed subset of $X$, one first extends $g$ to a continuous bounded function on $X$, then one extends this continuously over $\beta X$ and, finally, one takes the restriction of the latter function to $f^{-1}\left(K_{i}\right)$. This results in the desired extension of $g$ and the lemma is proved.

Now we are in a position to complete the proof of the theorem. Suppose $W$ belongs to $\mathscr{R}(X)$, that is, $W$ is a remainder of $X$. Then $\beta X-X$ maps continuously onto $W$ and hence, by Lemma 7 , the space $W$ is a continuous image of $\bigvee\left\{\beta A_{i}-A_{i}\right\}_{i=1}^{N}$. Now Theorem (2.11) of [2] states that the remainders of all ringed spaces are identical and by Lemma 8 each $A_{i}$ is a ringed space. We recall once again that a space is defined to be a ringed space if it is noncompact, locally compact, connected, locally connected, metric and has no two-point compactification. Since $R^{+}$is a ringed space, it follows in particular that $\mathscr{R}\left(R^{+}\right)=\mathscr{R}\left(A_{i}\right)$ for each $A_{i}$. Thus $\beta A_{i}-A_{i} \in \mathscr{R}\left(R^{+}\right)$and is therefore the continuous image of $\beta R^{+}-R^{+}$. Consequently, $W$ is the continuous image of the free union of $N$ copies of $\beta R^{+}-R^{+}$. 
Now suppose $W$ is a continuous image of $\bigvee\left\{Y_{i}\right\}_{i=1}^{N}$ where each $Y_{i}$ is homeomorphic to $\beta R^{+}-R^{+}$. Then $Y_{i} \in \mathscr{R}\left(A_{i}\right)$ and hence is a continuous image of $\beta A_{i}-A_{i}$. It follows that $\beta X-X=\bigvee\left\{\beta A_{i}-A_{i}\right\}_{i=1}^{N}$ maps continuously onto $\bigvee\left\{Y_{i}\right\}_{i=1}^{N}$ and hence onto $W$. However, since $X$ is locally compact, it follows from Theorem (2.1) of $[9$, p. 322] that $W$ belongs to $\mathscr{R}(X)$. This concludes the proof of the theorem.

Added in proof. Lemma 2 does, in fact, follow from a result already in the literature. On page 120 of Uniform spaces by J. R. Isbell (Math. Surveys, no. 12, Amer. Math. Soc., Providence, R. I., 1964), it is stated that a compact space is metric if it is a countable union of separable metrizable subspaces.

\section{REFERENCES}

1. N. Bourbaki, General topology. Part 2, Hermann, Paris; Addison-Wesley, Reading, Mass., 1966. MR $34 \# 5044 b$.

2. G. R. Blakley, J. Gerlits and K. D. Magill, Jr., A class of spaces with identical remainders, Studia Sci. Math. Hungar. (to appear).

3. J. Dugundji, Topology, Allyn and Bacon, Boston, Mass., 1966. MR 33 \#1824.

4. L. Gillman and M. Jerison, Rings of continuous functions, University Series in Higher Math., Van Nostrand, Princeton, N. J., 1960. MR 22 \#6994.

5. J. L. Kelley, General topology, Van Nostrand, Princeton, N. J., 1955. MR 16, 1136.

6. K. Kuratowski, Topologie. Vol. II, 3rd ed., Monografie Mat., Tom 21, PWN, Warsaw, 1961; English transl., Academic Press, New York; PWN, Warsaw, 1968. MR 24 \#A2958.

7. K. D. Magill, Jr., N-point compactifications, Amer. Math. Monthly 72 (1965), 1075-1081. MR $32 \# 3036$.

8. - Countable compactifications, Canad. J. Math. 18 (1966), 616-620. MR 33 \#6578.

9. - A note on compactifications, Math. Z. 94 (1966), 322-325. MR 34 \#3530.

10. - More on remainders of spaces in compactifications, Bull. Acad. Polon. Sci. 18 (1970), 449-451.

State University of New York at Buffalo, AMHERST, NeW YoRK 14226 